\title{
Dynamic fluid-structure-soil interaction: applications in earthquake engineering
}

\author{
A. Dimas, N. Bazeos, S. Bousias, T. C. Triantafyllou \\ \& D. L. Karabalis \\ Department of Civil Engineering, University of Patras, Greece
}

\begin{abstract}
A new discrete model is presented for the evaluation of the dynamic characteristics, i.e. eigenfrequencies and eigenmodes, of tanks of arbitrary shape and fill level. The accuracy and efficiency of the proposed methodology is demonstrated via a number of comparison studies. The above discrete model is combined with structural and soil simulation models for the efficient dynamic analysis of 3-D tanks under earthquake excitation. The obtained results are in excellent agreement to those obtained using detailed analytical and FEM models. Keywords: discrete sloshing model, arbitrary geometry, arbitrary fill level, seismic excitation, dynamic fluid-structure-soil interaction.
\end{abstract}

\section{Introduction}

The safe keeping and uninterrupted flow of liquids or liquid-like materials is of crucial and multifold importance to the industrialized world. Therefore, the interest on the seismic behaviour of modern structures used for storage of such materials has remained strong since the pioneering work of Lamb [1] and is periodically reinforced by the disruption caused by several seismic events, e.g. the 1964 Alaska earthquake. A simple, but accurate and efficient, methodology for the estimation of the hydrodynamic pressures exerted on the walls of a tank, was proposed in the pioneering work of Housner [2]. In these studies on nondeformable vertical prismatic tanks resting on rigid foundations, the solution describing the total hydrodynamic pressure was decomposed into two discrete parts: the "impulsive" and the "convective". The impulsive pressure component is due to a portion of the liquid accelerating with the rigid tank, while the 
convective pressure is exerted by the sloshing motion oscillating at a fundamental frequency. The "impulsive-convective" pressure concept along with the findings of a number of follow up studies, as referenced below, lie at the basis of almost all recent design codes and guidelines, e.g. API Standard 650 [3], Eurocode 8 [4] and ASCE [5].

In addition to the dynamics of a rigid liquid container, a number of other issues, of special interest to structural engineering, have been studied since these early works, most notably: the interaction between the deformable tank walls and the contained liquid, e.g. Veletsos and Shivakumar [6], and the interaction between the supporting medium (soil) and the tank structure, including uplifting and anchoring effects, e.g. Natsiavas and Babcock [7]. In contrast to the vast body of publications concerning vertical prismatic tanks, only few works have appeared on other tank geometries, e.g. Patkas and Karamanos [8]. This extensive analytic and numerical effort is supported by several experimental studies, e.g., Abramson et al. [9]. A more complete list of references on this subject can be found in the review articles of Rammerstorfer and Scharf [10], Ibrahim et al. [11].

Finally, brief reference is made to numerical solutions obtained by application of the most popular numerical methods, i.e. the finite (FEM) and the boundary (BEM) element methods, since they provide the means for the solution of complicated geometries, boundary conditions and couplings between the liquid and solid domains. A comprehensive discussion and literature review on these matters can be found in Rizos and Karabalis [12].

A search through the literature leaves no doubt that the "impulsiveconvective" pressure concept is almost universally used for the analysis and design of vertical prismatic tanks. However, very little or no information exists regarding the application of this concept to tanks of other geometries while the distribution of the hydrodynamic pressure on the walls of tanks of non-prismatic geometry is almost completely missing. This is mainly due to the fact that the development of impulsive-convective spring-mass systems is based, in most cases, on analytical solutions and, thus, the development of such simple solutions for more complicated geometries and boundary conditions is precluded. This work proposes a simple numerical methodology for the computation of the impulsive-convective mass system that can be used in the seismic design of liquid containers of arbitrary shape and fill height. To this end, an eigenvalue analysis is used in conjunction with a simple fluid model which is readily accessible through most of the commercially available general purpose FEM programs, e.g. ANSYS [13]. Even though a FEM analysis can handle a variety of complicated fluid and structural models, as well their various interactions, in an effort to concentrate on the influence of the geometry and to illustrate the generality of the proposed methodology, the fluid flow model used in this work is linear (small free surface amplitude) and inviscid (slip wall condition), while the tank walls are assumed rigid. 


\section{Numerical formulation}

The eigenvalue analysis of a fluid volume can be routinely performed, following an appropriate FEM discretization, by most of the available FEM general purpose programs and results such as the eigenfrequencies $\omega_{i}$ and the corresponding eigenvectors $\{\varphi\}_{\mathrm{i}}(\mathrm{i}=1,2, \ldots, 3 \mathrm{~N})$ are obtained, where $\mathrm{N}$ is the total number of nodal points. However, it should be noted that only those modes corresponding to a vertical motion of the free surface are of interest and retained for further analysis.

The total hydrodynamic pressure, applied on the tank walls, during a horizontal seismic excitation, can be decomposed into an impulsive part, associated with the fluid motion that has zero relative acceleration with respect to the tank, and a convective part, associated with the fluid sloshing motion. Likewise, the total horizontal force applied on the tank due to the fluid motion can be decomposed into an impulsive and a convective component. The sloshing motion can be represented as a superposition of few eigenmodes of the fluid motion. In general, each eigenmode generates a hydrodynamic wall pressure of unique distribution, but not all of these modes contribute to the development of a nonzero horizontal force on the tank. For example, for a vertically axisymmetric tank, the eigenmodes, which are responsible for the generation of a nonzero horizontal hydrodynamic force and, thus, influence the horizontal motion of the tank structure, are only those that exhibit free-surface antisymmetry with respect to a plane parallel to the tank axis of symmetry. These are the eigenmodes of interest in this work and hereafter are referred as "sloshing modes". The discrete masses $M_{C n}$, associated with the sloshing modes $\mathrm{n}=1, \ldots, \infty$, are called convective. The convective masses and the impulsive mass $M_{I}$, i.e. the remaining portion of the total mass moving in synchronism with the tank, are related via the principle of conservation of mass as

$$
M_{L}=M_{I}+\sum_{n=1}^{\infty} M_{C n}
$$

where $M_{L}$ stands for the entire liquid mass. The instantaneous value of the total horizontal hydrodynamic force or base shear, $F$, applied on the tank due to the liquid motion, can be computed as [6]

$$
F(t)=M_{I} \ddot{x}(t)-\sum_{n=1}^{\infty} M_{C n} \omega_{n}^{2} u_{n}(t)
$$

where $\ddot{x}(t)$ is the acceleration of the tank structure and $u_{n}(t)$ is the response of a single degree-of-freedom system, with frequency $\omega_{n}$,which is computed using as forcing input the acceleration of the tank. In view of the form of eqn (2), the concept of an oscillator with multiple degrees-of-freedom is employed in this work. Therefore, the convective mass $M_{C n}$ of each sloshing mode is equal to an effective modal mass computed as, e.g. Chopra [14],

$$
M_{C n}=\left(L_{C n}\right)^{2} / M_{n}
$$


where $M_{n}=\{\phi\}_{n}^{T}[M]\{\phi\}_{n}$ is the generalized mass of eigenmode $n$, and $L_{C n}=\{\phi\}_{n}^{T}[M]\{I\}$, with $\{I\}$ being the unit vector in the direction of the seismic excitation. A schematic representation of a convective-impulsive system, for a tank of arbitrary shape under horizontal seismic excitation, is shown in fig. 1 where the convective portion of the liquid is substituted by an equivalent system of masses and springs, while the impulsive portion reduces to a single mass rigidly connected to the tank wall.

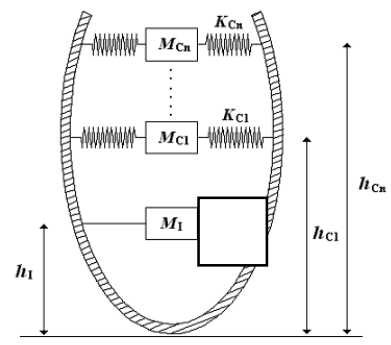

Figure 1: Impulsive-convective model for the representation of fluid motion.

On the basis of the previous discussion, the spring constants for each convective mass can be calculated as

$$
K_{C n}=\omega_{n}^{2} M_{C n} .
$$

The position height, $h_{C n}$ or $h_{n}$, of each mass depends on the convectiveimpulsive pressure distribution on the tank wall. For vertical prismatic tanks the position heights are available in analytic form, e.g. Refs [2,11]. For a spherical tank or a horizontal cylinder of diameter $D$, all discrete masses should be placed at the geometrical center, i.e. $h_{C n}=h_{n}=\mathrm{D} / 2$, since all resultant forces due to fluid pressure are applied at the geometrical center of the container. For arbitrary tank geometries, the corresponding heights can be calculated via a straightforward numerical integration of the fluid pressure distribution and subsequent positioning of the resultant force using standard procedures, e.g. Ref. [6].

Similarly, the total hydrodynamic pressure distribution on the tank wall can be decomposed, into an impulsive part, a convective part, and an axisymmetric part, associated with the axisymmetric eigenmodes of the fluid motion which do not contribute to the total horizontal force, as

$$
p(\theta, \varphi, t)=p_{I}(\theta, \varphi, t)+\sum_{n=1}^{\infty} p_{C n}(\theta, \varphi, t)+\sum_{m=1}^{\infty} p_{A m}(\theta, t)
$$

where $\theta$ and $\varphi$ are the azimuth and meridian angles, respectively, as shown in fig. $2, p_{I}$ is the impulsive pressure, $p_{C n}$ is the convective pressure due to sloshing 


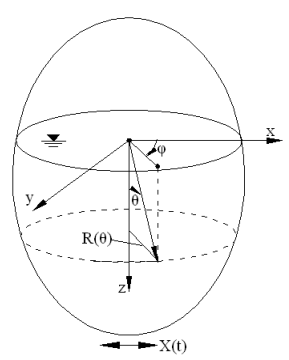

Figure 2: $\quad$ Coordinate system for axisymmetric (with respect to axis $z$ ) tanks.

mode $n$ and $p_{A m}$ is the axisymmetric pressure due to axisymmetric mode $m$. For vertically axisymmetric tanks under horizontal seismic excitation, only the sloshing modes are excited and the axisymmetric pressure is zero. The impulsive pressure is given by the expression

$$
p_{I}(\theta, \varphi, t)=C_{I}(\theta) \rho R(\theta) \cos (\varphi) \ddot{x}(t)
$$

while the convective pressure, for each sloshing mode $n$, by the expression

$$
p_{C n}(\theta, \varphi, t)=-C_{C n}(\theta) \omega_{n}^{2} \rho R(\theta) \cos (\varphi) u_{n}(t)
$$

with $C_{I}$ and $C_{C n}$ being dimensionless pressure profile functions which depend on the tank fill height. The computation of $C_{I}$ and $C_{C n}$ is based on a series of dynamic mode superposition analyses. Thus, for any fill height, the response of the fluid-structure FEM model to an arbitrary seismic excitation is computed taking into consideration only the n-th sloshing mode. The resulting hydrodynamic pressure distribution is equal to $p_{C n}$, and, therefore, the profile functions $C_{C n}$ can be computed using eqn (7). Then, a time domain simulation is performed, based on the same seismic excitation as in the dynamic mode superposition analysis, and the total hydrodynamic pressure distribution $p$ is obtained. Finally, the impulsive pressure distribution $p_{I}$ results from eqn (5) and the corresponding pressure profile function $C_{I}$ from eqn (6).

\section{Numerical example}

A series of numerical studies have been performed for the validation of the accuracy and efficiency of the proposed methodology. Due to lack of space, only an example pertaining to a seismic analysis of a spherical tank is presented in this work. However, the interested reader can find a series of related results in Drosos et al. [15], Drosos [16] and Drosos [17].

The spherical tank under investigation is shown in fig. 3 . Its diameter is $20 \mathrm{~m}$, its equator is located $12.5 \mathrm{~m}$ above the foundation, and contains a liquid with mass density $\rho=522 \mathrm{Kg} / \mathrm{m}^{3}$. The supporting system of 11 columns and 11 pairs of diagonal braces is resting on a circular foundation ring of external diameter 
$20 \mathrm{~m}$, approximately, which, in turn, rests on a category $\mathrm{C}$ soil medium [4]. Two models are considered for comparison purposes: (a) a detailed FEM where the fluid has been simulated by a mesh of 3D elements (ANSYS-FLUID80) and (b) a simplified model where the mass of the liquid has been decomposed into impulsive and convective parts in full accordance with eqns (1)-(7). The results of this decomposition, as used in the simplified model, are listed in table 1. However, details about the geometry, soil-structure interaction constants, material properties, etc., can be found in [15-17].

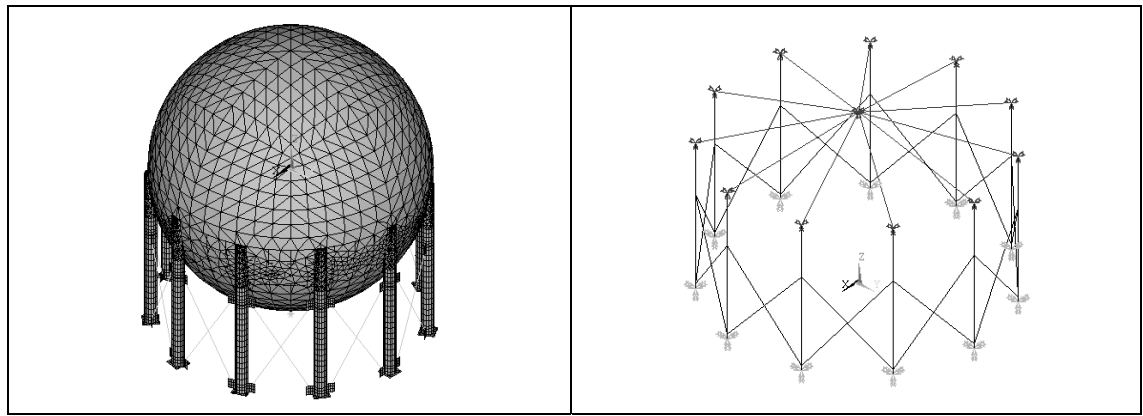

Figure 3: Models of spherical tanks: (a) a detailed FEM model and (b) a simplified model.

Table 1: $\quad$ Impulsive mass and convective mass and spring constants.

\begin{tabular}{|c|c|c|}
\hline & \multicolumn{2}{|c|}{ Fill level } \\
\hline & $50 \%$ & $100 \%$ \\
\hline $\begin{array}{c}\text { Impulsive mass }+ \text { mass } \\
\text { of steel shell } \\
(\mathrm{KNsec} / \mathrm{m})\end{array}$ & $445.6+394.5$ & $2186.7+394.5$ \\
\hline $\begin{array}{c}\text { Convective mass } M_{C l} \\
\left(\mathrm{KNsec}^{2} / \mathrm{m}\right)\end{array}$ & 621.4 & 0 \\
\hline $\begin{array}{c}\text { Spring constant } K_{C l} \\
(\mathrm{KN} / \mathrm{m})\end{array}$ & 954 & -- \\
\hline
\end{tabular}

With regard to the constants of table 1 it should be noted: (a) Only the first convective mode has a non-negligible effect on the dynamic behavior of this tank, and is used in the following, (b) the impulsive mass is rigidly attached to center of the sphere along with the mass of the steel shell, (c) the convective mass $M_{C l}$ is attached to the center of the sphere via the corresponding spring $K_{C l}$, 
(d) obviously no sloshing occurs in a full tank and thus the corresponding convective mass is zero.

The eigenvalue analysis of the above two models is shown in table 2 . Three levels of liquid fill are investigated: (a) empty tank, (b) half full tank, and (c) full tank. For all three cases two types of boundary conditions are considered: (i) the structure is fixed at its foundation, and (ii) soil-structure interaction (SSI) via the usage of spring and dashpot elements appropriate for a ring foundation [17]. The most important eigenvalues of the two models are listed along with the corresponding percentage of participating mass. Apparently there is a very good agreement between the results obtained by the two models. The results for the empty tank indicate that the two models are virtually equivalent with regard to their masses and stiffnesses. The results for the two fill levels, i.e. $50 \%$ and $100 \%$, also serve as a proof that the impulsive and convective masses computed by the proposed model can represent the fluid-structure model in an effective and accurate way.

Table 2: $\quad$ Results of eigenvalue analysis of FEM and simplified impulsiveconvective models of spherical tank.

\begin{tabular}{|c|c|c|c|c|c|}
\hline \multirow[b]{2}{*}{ Fill } & \multirow{2}{*}{$\begin{array}{l}\text { Support } \\
\text { conditions }\end{array}$} & \multicolumn{2}{|c|}{ Simplified model } & \multicolumn{2}{|c|}{ FEM model } \\
\hline & & $\begin{array}{l}\text { Period } \\
\mathrm{T}(\mathrm{sec})\end{array}$ & $\begin{array}{c}\text { Participating } \\
\text { mass }(\%)\end{array}$ & $\begin{array}{l}\text { Period } \\
\mathrm{T}(\mathrm{sec})\end{array}$ & $\begin{array}{c}\text { Participating } \\
\text { mass }(\%)\end{array}$ \\
\hline \multirow{2}{*}{$0 \%$} & Fixed & 0.250 & 99.10 & 0.242 & 96.80 \\
\hline & SSI & 0.185 & 63.70 & 0.185 & 60.30 \\
\hline \multirow{2}{*}{$50 \%$} & Fixed & 0.361 & 57.70 & 0.353 & 57.20 \\
\hline & SSI & 0.399 & 54.00 & 0.392 & 56.60 \\
\hline \multirow{2}{*}{$100 \%$} & Fixed & 0.630 & 98.70 & 0.592 & 83.60 \\
\hline & SSI & 0.690 & 55.00 & 0.649 & 87.00 \\
\hline
\end{tabular}

\section{Conclusions}

A simple and computationally-effective model has been proposed for the simulation of sloshing liquids in tanks of arbitrary shape. The methodology for the computation of the convective and impulsive masses and the associated spring constants is based on standard FEM analyses available in almost all commercially available finite element software. Seismic analyses of elevated spherical tanks, simulating realistic structures, reveal the computational efficiency and accuracy of the proposed models. Obviously, the use of the proposed discrete models results in a dramatic reduction of the size of the dynamic model. Thus, it can become a useful tool for quick, yet accurate, analyses in the design office. 


\section{References}

[1] Lamb, H., Hydrodynamics, Cambridge University Press, Cambridge, 1932.

[2] Housner, G.W., Dynamic pressures on accelerated fluid containers, Bulletin of the Seismological Society of America, 47, pp. 15-35, 1957.

[3] American Petroleum Institute, Seismic design of storage tanks - Appendix E: Welded Steel Tanks for Oil Storage (API Standard 650), Washington, D.C., 1995.

[4] Comité Européen de Normalization, Eurocode 8, Part 4: Silos, tanks and pipelines (Annex A), CEN ENV-1998-4, Brussels, 1998.

[5] ASCE Committee on Gas and Liquid Fuel Lifelines (Technical Council on Lifeline Earthquake Engineering), Guidelines for the seismic design of oil and gas pipeline systems, New York, 1984.

[6] Veletsos, S.A. and Shivakumar, P., Tanks containing liquids and solids (Chapter 15). Computer Analysis and Design of Earthquake Resistant Structures - A Handbook, eds D.E. Beskos and S.A. Anagnostopoulos, Computational Mechanics Publications, Southampton, 1984.

[7] Natsiavas, S. \& Babcock, C.D., Behavior of unanchored fluid-filled tanks subjected to ground excitation, Journal of Applied Mechanics, 55, pp. 654659, 1988.

[8] Patkas, L.A. and Karamanos, S.A., Variational Solutions of Liquid Sloshing in Horizontal-Cylindrical and Spherical Containers, Journal of Engineering Mechanics (ASCE), 133, pp. 641-655, 2007.

[9] Abramson, H.N., The dynamic behavior of liquids in moving containers (Southwest Research Institute), NASA SP-106, 1966.

[10] Rammerstorfer, F.G. and Scharf, K., Storage tanks under earthquake loading, Applied Mechanics Reviews (ASME), 43, pp. 261-282, 1990.

[11] Ibrahim, R.A., Pilipchuk, V.N. and Ikeda, T., Recent advances in liquid sloshing dynamics, Applied Mechanics Reviews (ASME), 54, pp. 133-177, 2001.

[12] Rizos, D.C. and Karabalis, D.L., Soil-Fluid-Structure Interaction (Chapter 9). Wave Motion in Earthquake Engineering, eds E. Kausel and G.D. Manolis, WIT PRESS, Southampton, 2000.

[13] Swanson Analysis Systems, Inc., ANSYS, User's Manual for Revision 5.0, Procedures, Houston, PA, 1992.

[14] Chopra, A.K., Dynamics of Structures, Prentice Hall, New Jersey, 1995.

[15] Drosos, G.C., Dimas, A.A. and Karabalis, D.L., Discrete models for seismic analysis of liquid storage tanks of arbitrary shape and fill height, Journal of Pressure Vessel Technology, 130, pp. 1-12, 2008.

[16] Drosos, G.C., The effect of sloshing on spherical tanks (in Greek), MS Master Thesis, Department of Civil Engineering, University of Patras, 2005.

[17] Drosos, J.C., A study of seismic protection systems for spherical tanks (in Greek), MS Master Thesis, Department of Civil Engineering, University of Patras, 2005. 